\title{
ILSHR Rumor Spreading Model by Combining SIHR and ILSR Models in Complex Networks
}

\author{
Adel Angali \\ Department of Computer Engineering, Liyan Institute of Education, Bushehr, Iran \\ E-mail: angali.adel@gmail.com \\ Musa Mojarad* \\ Department of Computer Engineering, Firoozabad Branch, Islamic Azad University, Firoozabad, Iran \\ E-mail: m.mojarad@iauf.ac.ir
}

\author{
Hassan Arfaeinia \\ Department of Computer Engineering, Liyan Institute of Education, Bushehr, Iran \\ E-mail: harfaeinia@gmail.com
}

Received: 27 July 2021; Accepted: 12 October 2021; Published: 08 December 2021

\begin{abstract}
Rumor is an important form of social interaction. However, spreading harmful rumors can have a significant negative impact on social welfare. Therefore, it is important to examine rumor models. Rumors are often defined as unconfirmed details or descriptions of public things, events, or issues that are made and promoted through various tools. In this paper, the Ignorant-Lurker-Spreader-Hibernator-Removal (ILSHR) rumor spreading model has been developed by combining the ILSR and SIHR epidemic models. In addition to the characteristics of the lurker group of ILSR, this model also considers the characteristics of the hibernator group of the SIHR model. Due to the complexity of the complex network structure, the state transition function for each node is defined based on their degree to make the proposed model more efficient. Numerical simulations have been performed to compare the ILSHR rumor spreading model with other similar models on the Sina Weibo dataset. The results show more effective ILSHR performance with 95.83\% accuracy than CSRT and SIR-IM models.
\end{abstract}

Index Terms: Complex networks, rumor spreading, SIHR model, ILSR model, ILSHR model.

\section{Introduction}

Social networks have become one of the most important information media in the world and studying the phenomena of spreading rumors on social networks is useful for understanding the inherent laws of publishing behavior [1]. Rumors play an essential role in social interaction and its spread has a significant impact on human life. Examining the dynamic mechanism of spreading rumors at various events can help social media managers in controlling and guiding public opinion. Malicious rumors can violate the interests of others, disrupt public order, and threaten social stability. For crisis communicators, it is important to understand when and how rumors should be controlled. [2]

Spreading rumors, as an important form of social communication, has generated widespread interest over the past few decades. In 1965, Deli and Kendall introduced the classic DK rumor model, which led to further research into rumor spreading. Then, in 1973, Maki and Thomson upgraded the DK to the MK. based on these ground breaking work, many rumor-spreading models have been developed and studied by researchers. Note that the rumor-mongering process of most papers does not take into account the impact of complex network topologies [3]. However, with the rapid advancement of technology, complex networks such as the Internet and the World Wide Web have permeated people's lives.

The users of complex networks have different roles in the process of spreading rumors, and the impact of these plans on spreading rumors should be considered. A user may, depending on their knowledge, publish a rumor when confronted with it, or they may not publish it or review it. If the user does not spread the rumor but the users around him do so, the user is more likely to become a broadcaster to spread the rumor. In general, users of a complex network can be divided into regular users and important users for the issue of spreading rumors. Ordinary users have no knowledge and blindly follow others. However, important users have rich knowledge and do not easily become spread rumors. Suppose $\bar{k}$ is the average degree of all network nodes and $k_{i}$ is the degree of node $\mathrm{i}$. If $\bar{k}<k_{i}$, then node $i$ is an important node and otherwise a normal node [4].

The lack of restrictions on the sharing of information on social networks has led to the dissemination of 
information regardless of their validity. This information generates and spreads rumors. Different users play different roles in the process of spreading rumors depending on the level of knowledge. Therefore, the effect of differences between users' behavior and knowledge in spreading rumors should be considered. Based on this, in this paper, a new hybrid epidemic rumor propagation model based on the degree of each node is developed. SIR rumor Spreading model one of the most basic gossip models for user analysis over time. SIR was first used to model the spread of viral diseases and epidemics and then used to analyze the spread of rumors [5]. In this model, each person in the community can be in one of the Susceptible (S), Infected (I) and Recovered (R) groups at any time. The SIR model can predict the spread of the rumor in the future as well as its management and control. This paper introduces a new rumor model that combines ILSR and SIHR rumor models.

The continuation of the paper is as follows. Section 2 describes related work. The proposed rumor Spreading model is described in Section 3. In Section 4, the simulation results are presented and finally in Section 5, conclusions are presented.

\section{Literature Review}

So far, many methods have been proposed to detect and analyze the spread of rumors on complex networks. Here are some of the latest researches. Singh et al. (2019) proposed a new model analysis for spreading rumors on a social network based on the Mittag-Leffler law [8]. In this paper, the dynamics model of rumor spreading in a social network related to the Atangana-Baleanu derivative of incorrect order is studied.

Chen (2019) proposed the ILSCR rumor spreading model for analysis on emergency rumor control [9]. In this paper, a new rumor model for control analysis is considered, considering the possibility that distributors may experience a cooling off period before being removed and people move in a particular area. Spreading rumors in emergencies is suggested. Jiang et al. (2020) developed a two-step rumor model, susceptible, positive, negative, and recovered state (SPNR) for rumor analysis and inversion of emergencies in Sina Weibo [10]. This model considers the hysteria of official news and the general fluctuation mentality based on epidemic models.

Zhu and Wang (2020) examined the stability analysis of the SAIR rumor-mongering model with control strategies in online social networks [11]. In order to investigate the effect of silence enforcement on broadcasters, this paper proposes a rumor-spreading model with the function of enforcing silence on online social networks. Yang et al. (2020) introduced the LT1DT rumor spreading model as a linear threshold model with one direction state transition for modeling in complex networks [12]. In this paper, the issue of minimizing rumor spread on social networks is investigated and a new approach based on spreading dynamics is performed by LT1DT.

Islam et al. (2020) proposed the DLSTM rumor spreading model for rumor detection on the Sina Weibo social network [13]. This model includes Deep Recurrent Neural Network (DRNN) and data filtering for rumor prediction work. DRNN is a symmetric gossip network that performs better than the basic RNN architecture. In DLSTM, the posts are first filtered according to the user's followers, and then the posts are coded sequentially. Chen and Wang (2020) proposed a rumor model based on rumor validation, correlation, and population classification based on user behavior [14]. In this paper, it is suggested that people with different behaviors behave differently after hearing rumors. Thus, populations are divided into two types: radicals and fixed individuals.

Li et al. (2020) proposed a rumor-spreading model by considering individual activity and the mechanism of simultaneous rejection [15]. In this paper, the differences between individual activity and the nodes of rumor rejection are considered simultaneously. In addition, the local stability of the model equilibrium is determined by the RouthHurwitz criteria, and the global stability is proved by a geometric method based on the use of a higher degree generalization of the Benedixon criterion. Yang et al. (2020) proposed the dynamics of the rumor-spreading model with a skeptical mechanism in heterogeneous networks and bilingual environments [16]. In this paper, a new rumormongering model under a bilingual environment and heterogeneous networks is proposed, which thinks that exposure may become a diffuser at a set speed. In the first stage, the non-negativity and limitation of the solution for the rumormongering model are proved by reduction and proof. In the second stage, the global stability of the dominant balance of rumors is examined using the Lyapunov method and the principle of non-change of LaSalle.

Ai et al. (2021) proposed a CSRT rumor-spreading model based on a complex network [17]. This paper improves the traditional Barabasi-Albert scaleless network and presents a network topology model that conforms to the characteristics of social network sharing based on complex network theory as well as the actual characteristics of network sharing. Qiu et al. (2020) introduced the SIR rumor-spreading model with a social media penetration mechanism (SIR-IM) [18]. The SIR-IM model first considers the number of current players for the probability of release. It then uses the time function to describe how many people have been removed from the player over time.

\section{Proposed Method}

In this paper, a new rumor model is introduced, which is an extension of the SIHR and ILSR rumor models. This model is named ILSHR. The SIHR rumor spreading model places people in the community into four groups: Susceptible-Infected-Hibernating-Removed. Social network users in the ILSR model are also organized into four 
groups: Ignorant-Lurker-Spreader-Removal. However, we combine the two models into ILSHR, where we place users into five groups: ignorant-Lurker-Spreader-Hibernator-Removal. In the following, a series of semantic equations are presented to describe the dynamics of the rumor-spreading model. Then, the rules of rumor spread and state transfer functions in the proposed model are presented. The proposed state transfer functions analyze the network steady state based on the degree of each node and are derived from the model rules. These functions indicate how likely users from each group are to be transferred to other groups.

The word influence in Webster's university dictionary is defined as "the power or capacity of an individual or the factors that influence him in direct or indirect ways." Here, social impact is defined by researchers as a change in an individual's thoughts, feelings, attitudes, or behaviors that results from interacting with another person or group. In general, in the phenomenon of social influence, a person's behavior influences the behavior of his friends and through them the person's friends behave similarly. Thus, the phenomenon of influence shows a person's ability to persuade others to behave similarly to himself. In general, influential nodes are the same influential nodes in a social network, the number of which is often limited.

\subsection{Population density in the SIHR rumor spreading model}

The SIHR model was developed to solve the SIR model problem [19]. The SIR model does not take into account the assumption that an ignorant (infected) person may directly become a strict (removed) person. In addition, forgetfulness and reminder mechanisms are not considered in SIR. In the mechanism of forgetfulness, a person may spontaneously stop spreading rumors, and in the mechanism of reminder, a person may spontaneously republish rumors. The SIHR classifies people into four groups: Susceptible (S), Infected (I), Hibernating (H), and Removed (R). Fig. 1 shows the population density in the SIHR model. Here $\lambda$ is the rumor rate, $\beta$ is the rumor rejection rate, $\delta$ is the forgetfulness rate, $\xi$ is the spontaneous reminder rate, $\eta$ is the wake-up reminder rate and $\alpha$ is the hardening rate.

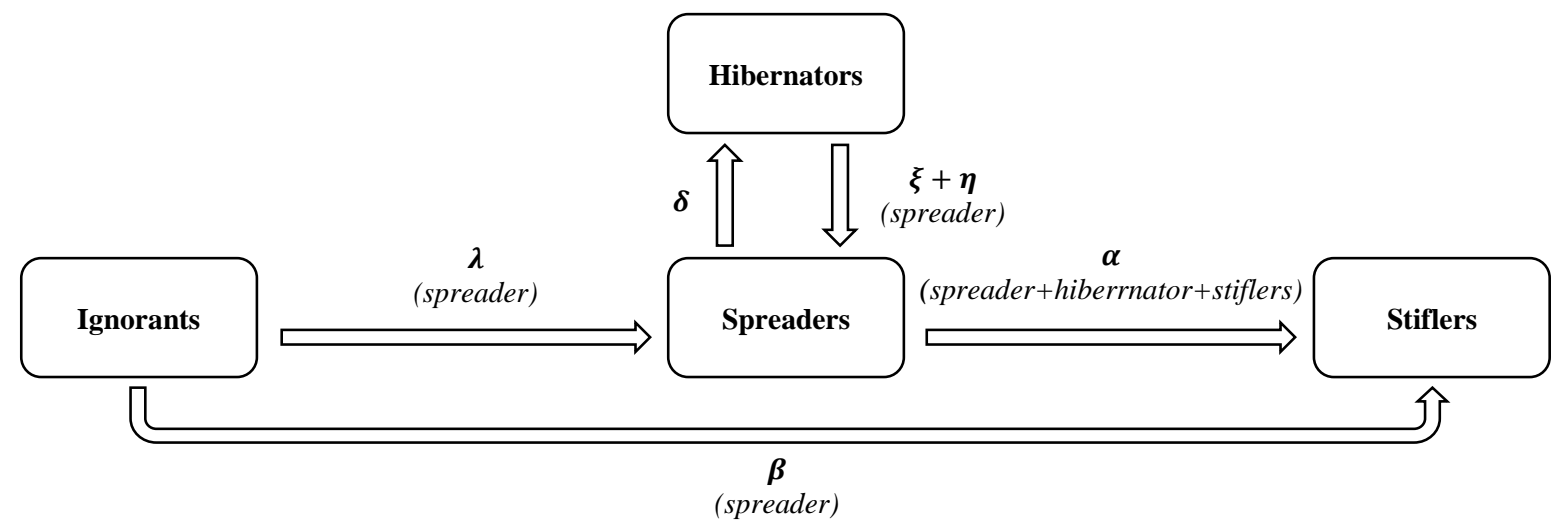

Fig.1. Population density in the SIHR model

In the SIHR model, $I(t), S(t), H(t)$, and $R(t)$ represent population densities, representing the infected, susceptible, hibernating, and removed, respectively. According to the rumor spreading process, the semantic equations of the SIHR model can be expressed as Eq. (1) to Eq. (4).

$$
\begin{gathered}
\frac{d I(t)}{d(t)}=-(\lambda+\beta) \bar{k} I(t) S(t) \\
\frac{d S(t)}{d(t)}=\lambda \bar{k} I(t) S(t)-\alpha \bar{k} S(t)[S(t)+H(t)+R(t)]-\delta S(t)+\xi H(t)+\eta \bar{k} H(t) S(t) \\
\frac{d H(t)}{d(t)}=\delta S(t)-\xi H(t)-\eta \bar{k} H(t) S(t) \\
\frac{d R(t)}{d(t)}=\beta \bar{k} I(t) S(t)+\alpha \bar{k} S(t)[S(t)+H(t)+R(t)]
\end{gathered}
$$

\subsection{Population density in the ILSR rumor spreading model}

The ILSR model divides individuals into four groups: Ignorant (I), Lurker (L), Spreader (S), and Removal (R) [20]. The ILSR has added a suspicious group to the SIR model, where it represents people who have heard the rumor but are skeptical and temporarily do not broadcast it. Fig. 2 shows the population density in the ILSR model. Here, $a_{1}$ is the rumor rate, $a_{2}$ is the non-disclosure rate, $\beta$ is the republishing rate, and $\delta$ is the hardening rate. 


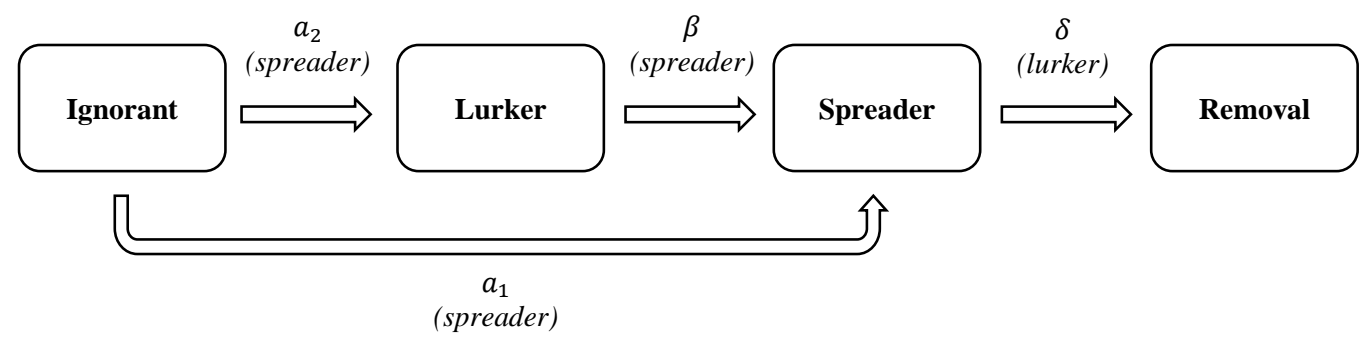

Fig.2. Population density in the SIHR model

In the ILSR model, $I(t), L(t), S(t)$, and $R(t)$ are the population densities that represent ignorant, lurker, spreader, and removal individuals, respectively. According to the rumor spreading process, the semantic equations of the ILSR model can be expressed as Eq. (5) to Eq. (8).

$$
\begin{gathered}
\frac{d I(t)}{d(t)}=-a_{1} I(t) S(t)-a_{2} I(t) S(t) \\
\frac{d L(t)}{d(t)}=a_{2} I(t) S(t)-\beta L(t) \\
\frac{d S(t)}{d(t)}=a_{1} I(t) S(t)+\beta L(t)-\delta S(t) \\
\frac{d R(t)}{d(t)}=\delta S(t)
\end{gathered}
$$

\subsection{Population density in the ILSHR rumor spreading model}

Based on previous studies, this research presents a modified rumor model from the SIR model that makes the rumor spreading process more realistic and obvious. In this paper, an extended model of SHIR and ILSR called ILSHR is presented. In this model, hibernation group has been added from SIHR to ILSR quadruple group. Therefore, users in the proposed model include five groups of Ignorant (I), Lurker (L), Spreader (S), Hibernator (H) and Removal (R). Fig. 3 shows the population density in the proposed rumor spreading model. Here, $a_{1}$ are temporary rumor failure rate, $a_{2}$ the rumor rate, $a_{3}$ the non-disclosure rate, $\beta$ the republishing rate, $\xi$ the self-reminder reminder rate, $\eta$ the wake-up reminder rate, $\delta_{1}$ the forgetting rate and $\delta_{2}$ the hardening rate. In addition to the characteristics of suspects, the proposed rumor model also takes into account the characteristics of hibernators. In the ILSHR model, $I(t), L(t), S(t), H(t)$, and $R(t)$ represent the population density, which represents the ignorant, lurker, spreader, hibernator, and removal individuals, respectively.

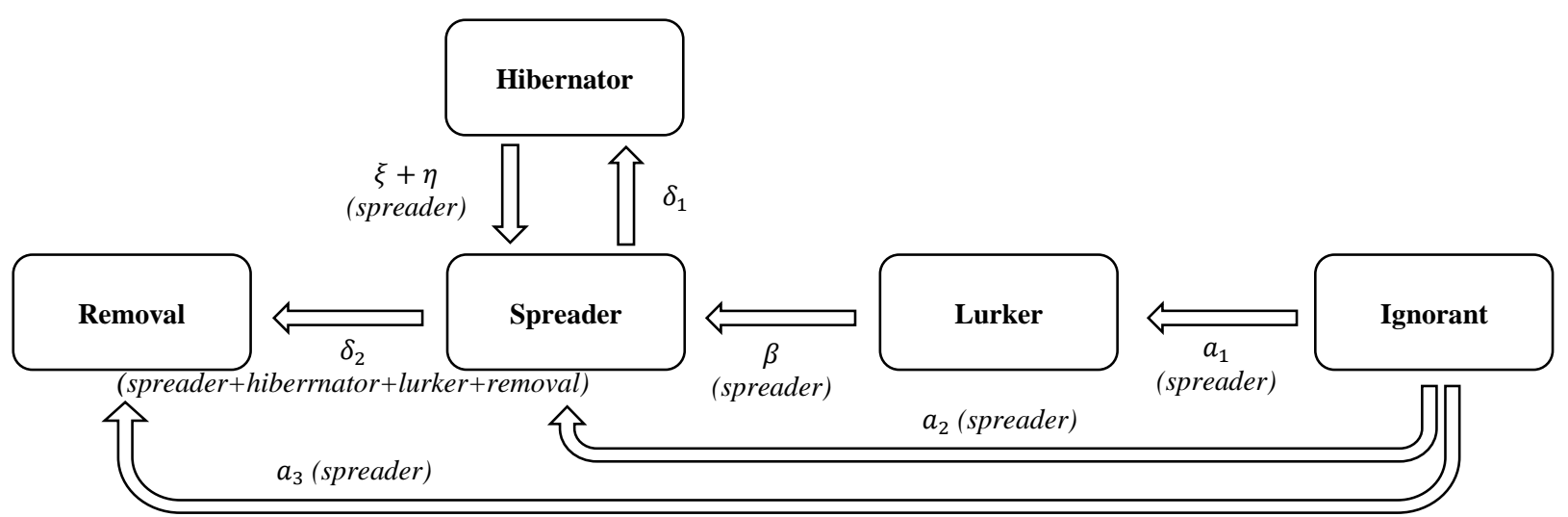

Fig.3. Population density in the ILSHR model follows:

Based on the rumor spreading model introduced in the ILSHR, the rumor spreading rules can be described as

1) By connecting ignorant people with spreaders; Ignorant people with $a_{1}$ probability become lurker people. Ignorant people also become spreaders people with $a_{2}$ probability and removal people with $a_{3}$ probability.

2) By connecting lurkers with spreaders; lurked individuals with a probability of $\beta$ become members of the spreader group. 
3) Spreaders with a probability of $\delta_{1}$ spontaneously become members of the hibernator group, which is known as the mechanism of forgetfulness.

4) People from the hibernator group are more likely to become spontaneous than the spreader group, which is known as the spontaneous recall mechanism.

5) By connecting hibernators with spreaders; people who hibernate with a probability of $\eta$ become spreaders, which is known as the wake-up call mechanism.

6) By connecting of spreaders with spreaders or hibernators or lurkers or removal; starter spreader with a probability of $\delta_{2}$ become members of the removal group.

According to the rumor spreading process, the semantic equations of the ILSHR model can be expressed as Eq. (9) to Eq. (13).

$$
\begin{gathered}
\frac{d I(t)}{d(t)}=-\left(a_{1}+a_{2}+a_{3}\right) I(t) S(t) \\
\frac{d L(t)}{d(t)}=a_{1} I(t) S(t)-\beta L(t) \\
\frac{d S(t)}{d(t)}=a_{2} I(t) S(t)+\beta L(t)-\delta_{1} S(t)-\delta_{2} S(t)[S(t)+H(t)+\mathrm{L}(\mathrm{t})+R(t)]+\xi H(t)+\eta H(t) S(t) \\
\frac{d H(t)}{d(t)}=\delta_{1} S(t)-\xi H(t)-\eta H(t) S(t) \\
\frac{d R(t)}{d(t)}=\alpha_{3} I(t) S(t)+\delta_{2} S(t)[S(t)+H(t)+\mathrm{L}(\mathrm{t})+R(t)]
\end{gathered}
$$

The rumor spread process in ILSHR starts with only one spreader. In this model, a society is considered with N individuals, so that the Eq. (14) is established.

$$
I(t)+L(t)+S(t)+H(t)+R(t)=1
$$

Therefore, the initial conditions in the proposed rumor spreading model are in the form of Eq. (15).

$$
I(0)=\frac{N-1}{N}, L(0)=0, S(0)=\frac{1}{N}, H(0)=0, \quad R(0)=0
$$

Based on the above discussion, the following state transfer functions for the ILSHR model are presented. These functions show how people from different groups are likely to become other groups. These probabilities include $a_{1}, a_{2}$, $a_{3}, \beta, \xi, \eta, \delta_{1}$ and $\delta_{2}$. The proposed state transfer functions perform the modeling work based on the degree of nodes (number of neighbors). $k_{i}$ refers to the degree of node $i$ and $\bar{k}$ refers to the average degree of all network nodes. $k_{i}>\bar{k}$ means that node $i$ is an important node in the network and $k_{i} \leq \bar{k}$ indicates that node $i$ is a normal node.

- The spreader node $i$ sends the rumor to the ignorant node $j$; The probability that the ignorant node $j$ becomes a node of the lurker or spreader or removal group can be calculated as Eq. (16) and Eq. (17).

$$
\begin{gathered}
\alpha_{f\left(k_{j}\right)}(i, j)=1-\frac{k_{j}}{\sum_{r \in \tau(i)} k_{r}} \\
f\left(k_{j}\right)= \begin{cases}1 & k_{j}>\bar{k} \\
2 & k_{j} \leq \bar{k} \\
3 & \frac{1}{2} k_{j}>\bar{k}\end{cases}
\end{gathered}
$$

Where, $\tau(i)$ is the list of neighbors of node $i$. Given the effect of the degree of node $j$, the conversion rates of the probabilities $a_{1}, a_{2}$ and $a_{3}$ can be calculated.

- Lurker node $j$ is affected by spreader node $i$; The probability that the lurker node $j$ becomes a node of the spreader group can be calculated as Eq. (18).

$$
\beta(i, j)=\frac{k_{i}}{k_{i}+k_{j}}
$$


- The probability that the spreader node $i$ becomes a lurker node or hibernator or removal or strict can be calculated as Eq. (19).

$$
\delta_{2}(i)=\frac{k_{i}+\sum_{r \in \gamma(i)} k_{r}+\sum_{r \in \zeta(i)} k_{r}+\sum_{r \in \sigma(i)} k_{r}}{k_{i}+\sum_{j \in \tau(i)} k_{j}}
$$

Where, $\gamma(i)$ is the list of spreader neighbors of node $i, \zeta(i)$ is the list of lurker neighbors of node $i$, and $\sigma(i)$ is the list of hibernating neighbors of node $i$.

- The probability of spontaneously converting spreader node $i$ to a node of hibernation group is constant and is shown as $\delta_{1}(i)$. This possibility arises from forgetting the spreader node.

- The probability of spontaneously converting the hibernation node $i$ to a node of the spreader group is constant and is shown as $\xi(i)$. This possibility arises from the recollection of the hibernation node.

- By connecting the hibernation node $i$ with the spreader node $j$; The probability that hibernation node $i$ becomes a node of the spreader group can be calculated as Eq. (20). This possibility arises from the reminder of the awakening of the hibernation node.

$$
\eta(i, j)=\frac{k_{j}+\sum_{r \in \tau(k)} k_{r}}{k_{i}+\sum_{r \in \tau(i)} k_{r}}
$$

\section{Simulation Results}

In this research, Sina Weibo social network data set has been used to compare and evaluate the proposed model. Details of this dataset can be found at http://alt.qcri.org/ wgao/data/rumdect.zip. The proposed model is simulated with MATLAB R2019a software and on an Asus laptop with an Intel Core i5 CPU at 3.2GHz and 8GB of RAM and Windows 10 64-bit operating system. In this section, time simulations are performed to observe user behavior in the population density of these models. To do this, a hypothetical network of $N=6 \times 10^{7}$ nodes for 100 days and every 6 hours per day is considered [21]. For convenience, the average degree of nodes is set to $\bar{k}=1$ [22].

This article analyzes the spread of rumors based on user posts in the Cena Weibo social network dataset. Sina Weibo social network is related to China tourism. For rumor detection and prediction, pre-processing of the Cena Weibo dataset is performed. The available dataset includes a number of Weibo posts with rumor and non-rumor tags. Posts are sent by users, where a user can have one or more posts. For rumor detection work, several features related to spreading rumors are extracted from users' posts. To do this, user posts are segmented using Jieba natural language processing software. Then, all the qualitative values in the data set become quantitative.

Fig. 4 shows the spreading process in the population density of the ILSHR model. In addition, we have shown population densities in the SIHR and ILSR models in Fig. 5 and 6, respectively.

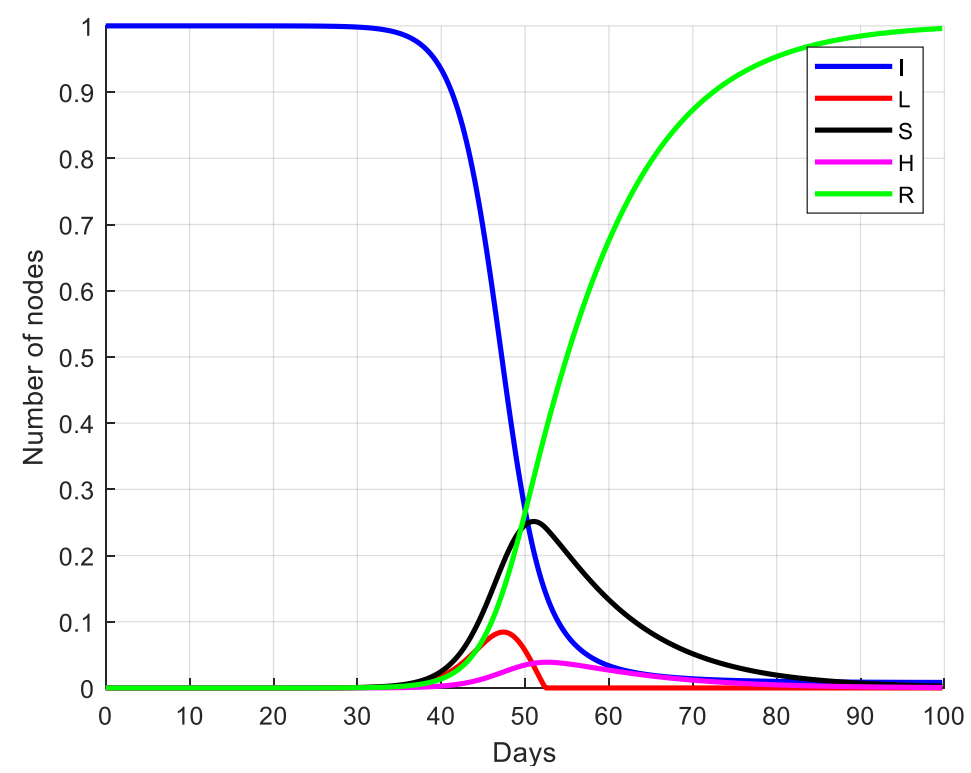

Fig.4. Population density in the ILSHR model over time 


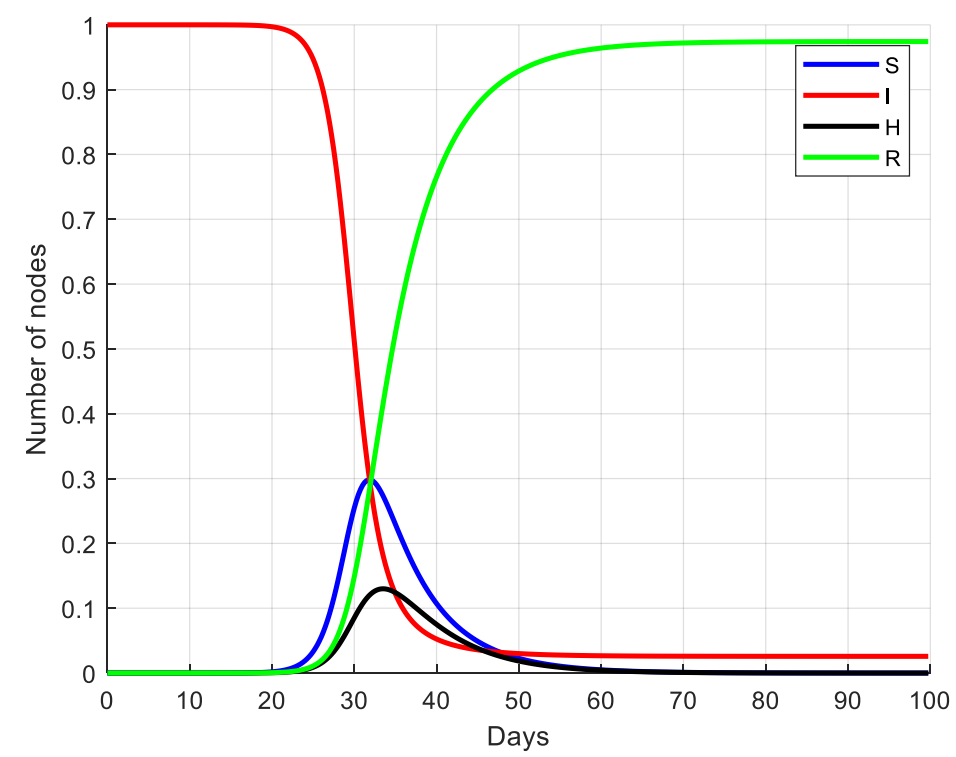

Fig.5. Population density in the SIHR model over time

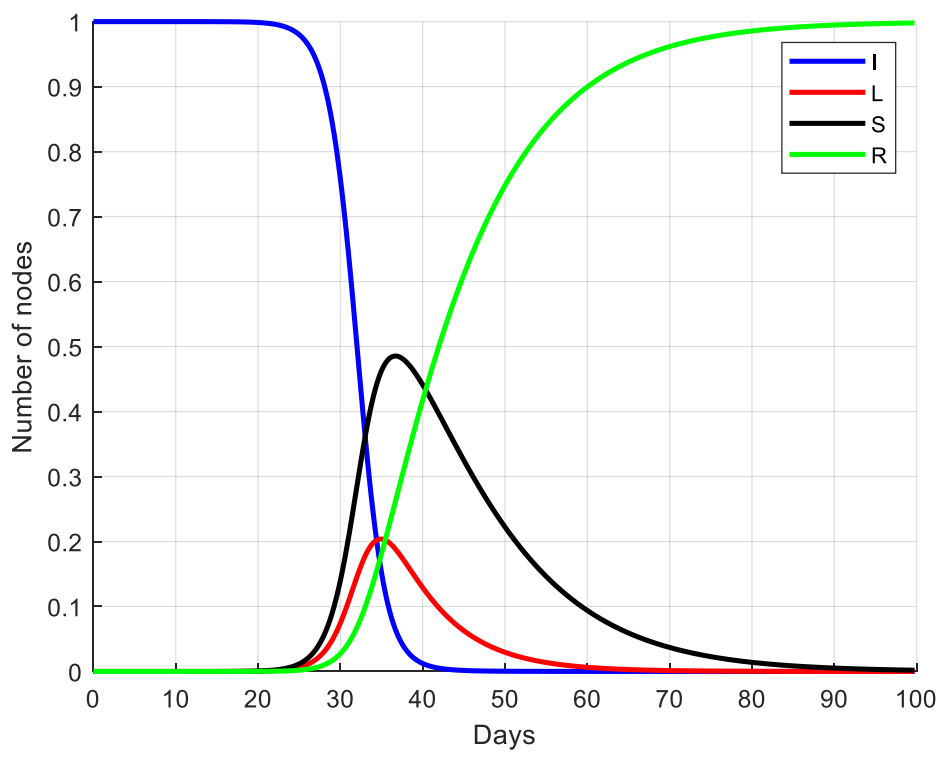

Fig.6. Population density in the ILSR model over time

The overall process of ILSHR model is similar to the SIHR and ILSR models, where the number of spreaders and removals in this process is constantly decreasing and increasing, respectively, to bring the system to a stable state. The process of changing the number of lurkers and hibernators is similar to that of the removal ones, which initially increases and then decreases to zero. The basic parameters for simulating the ILSHR model are as follows.

$$
\begin{gathered}
S(0)=\frac{1}{6 \times 10^{7}}, I(0)=\frac{6 \times 10^{7}-1}{6 \times 10^{7}}, L(0)=0, H(0)=0, R(0)=0, \alpha_{1}=0.5, \alpha_{2}=0.3, \\
\alpha_{3}=0.2, \beta=0.25, \delta_{1}=0.1, \delta_{2}=0.25, \xi=0.5, \eta=0.5
\end{gathered}
$$

For this Weibo post, 14 features are extracted from the Sina Weibo dataset. Therefore, a two-class classification model is needed to detect rumors. This model can be used to predict and detect rumors. Here the three classification models SVM, MLP (with a hidden layer) and ID3 are compared for rumor detection and modeling. Finally, it can be seen that the accuracy of SVM detection is better than other models, although the results of MLP neural network are also promising. Table 1 shows the results of this comparison. 
Table 1. Comparison of different classification models in rumor detection

\begin{tabular}{|c|c|c|c|c|}
\hline Models & Accuracy $(\%)$ & Precision & Recall (\%) & F-measure (\%) \\
\hline SVM & 95.83 & 96.95 & 94.38 & 95.665 \\
\hline MLP & 95.12 & 96.70 & 94.26 & 95.46 \\
\hline ID3 & 93.50 & 94.84 & 95.94 & 95.39 \\
\hline
\end{tabular}

The results show that SVM with $95.83 \%$ rumor accuracy has the best performance compared to other models. The MLP has since detected $95.12 \%$ of the rumors. In general, SVM offers better performance for detecting rumors.

Early detection of rumors (in the early stages of publication) is very important. Here, we categorize the Sina Weibo dataset based on post time at the first 4, 8, 12, 24, 48, 72, and 96 hours, and then compare different models for rumor detection in the early stages of publication. The results of this comparison for the proposed method and models of CSRT [17] and SIR-IM [18] are given in Table 2. The results show the superiority of the proposed model compared to CSRT and SIR-IM models in all time gaps. The average results show that the proposed model has achieved $92.25 \%$ accuracy in the first 12 hours of posting.

Table 2. Comparison of different models in rumor detection based on rumor publication time

\begin{tabular}{|c|c|c|c|c|c|}
\hline Models & 12 & 24 & 48 & 72 & 96 \\
\hline CSRT & 91.45 & 93.50 & 94.09 & 94.26 & 94.00 \\
\hline SIR-IM & 92.08 & 94.21 & 94.32 & 94.63 & 94.11 \\
\hline ILSHR & 92.25 & 94.18 & 94.80 & 94.86 & 94.35 \\
\hline
\end{tabular}

\section{Conclusion}

Recent studies on the process of gossiping or disseminating information on complex networks show that, contrary to traditional understanding, people who participate in spreading gossip on a network do not always receive gossip from their neighbors. In this paper, in order to analyze the spread of rumors in complex networks, a new model of spreading rumors called ILSHR was presented. This developed model is the SIHR and ILSR rumor spread models. ILSHR divides users into important and normal due to different characteristics for understanding people's behavior about rumors. In addition, the ILSHR divides the population into five groups: ignorant-lurker-spreader-hibernator-removal. In addition, the ILSHR model is analyzed and a new state transfer function based on the degree of different nodes is designed to study the law of rumor spreading in complex networks. This model has been confirmed by simulation on the Sina Weibo network. For future work, it is suggested to use the features of new rumor mills such as $2 \mathrm{SIH} 2 \mathrm{R}$ or ISRC to provide better population diversity based on them.

\section{References}

[1] Rezaeipanah, A., Ahmadi, G., \& Matoori, S. S. (2020). A classification approach to link prediction in multiplex online egosocial networks. Social Network Analysis and Mining, 10(1), 1-16.

[2] Kosfeld, M. (2005). Rumours and markets. Journal of Mathematical Economics, 41(6), 646-664.

[3] Li, J., Jiang, H., Yu, Z., \& Hu, C. (2019). Dynamical analysis of rumor spreading model in homogeneous complex networks. Applied Mathematics and Computation, 359, 374-385.

[4] Li, J., Jiang, H., Yu, Z., \& Hu, C. (2019). Dynamical analysis of rumor spreading model in homogeneous complex networks. Applied Mathematics and Computation, 359, 374-385.

[5] Prathamesh Churi, N. T. Rao, " Teaching Cyber Security Course in the Classrooms of NMIMS University ", International Journal of Modern Education and Computer Science(IJMECS), Vol.13, No.4, pp. 1-15, 2021.DOI: 10.5815/ijmecs.2021.04.01

[6] Ma, C. (2017). Dynamical analysis of rumor spreading model with impulse vaccination and time delay. Physica A: Statistical Mechanics and its Applications, 471, 653-665.

[7] Cheng, Y. (2019). Dynamical analysis of a IWSR rumor spreading model with considering the self-growth mechanism and indiscernible degree. Physica A: Statistical Mechanics and its Applications, 536, 120940.

[8] Singh, J. (2019). A new analysis for fractional rumor spreading dynamical model in a social network with Mittag-Leffler law. Chaos: An Interdisciplinary Journal of Nonlinear Science, 29(1), 013137.

[9] Chen, G. (2019). ILSCR rumor spreading model to discuss the control of rumor spreading in emergency. Physica A: Statistical Mechanics and its Applications, 522, 88-97.

[10] Jiang, G., Li, S., \& Li, M. (2020). Dynamic rumor spreading of public opinion reversal on Weibo based on a two-stage SPNR model. Physica A: Statistical Mechanics and its Applications, 558, 125005.

[11] Zhu, L., \& Wang, B. (2020). Stability analysis of a SAIR rumor spreading model with control strategies in online social networks. Information Sciences, 526, 1-19.

[12] Yang, L., Li, Z., \& Giua, A. (2020). Containment of rumor spread in complex social networks. Information Sciences, 506, 113130.

[13] Islam, M. R., Liu, S., Wang, X., \& Xu, G. (2020). Deep learning for misinformation detection on online social networks: a 
survey and new perspectives. Social Network Analysis and Mining, 10(1), 1-20.

[14] Chen, X., \& Wang, N. (2020). Rumor spreading model considering rumor credibility, correlation and crowd classification based on personality. Scientific reports, 10(1), 1-15.

[15] Li, R., Li, Y., Meng, Z., Song, Y., \& Jiang, G. (2020). Rumor Spreading Model Considering Individual Activity and Refutation Mechanism Simultaneously. IEEE Access, 8, 63065-63076.

[16] Yang, S., Jiang, H., Hu, C., Yu, J., \& Li, J. (2020). Dynamics of the rumor-spreading model with hesitation mechanism in heterogenous networks and bilingual environment. Advances in Difference Equations, 2020(1), 1-21.

[17] Ai, S., Hong, S., Zheng, X., Wang, Y., \& Liu, X. (2021). CSRT rumor spreading model based on complex network. International Journal of Intelligent Systems, 36(5), 1903-1913.

[18] Qiu, L., Jia, W., Niu, W., Zhang, M., \& Liu, S. (2020). SIR-IM: SIR rumor spreading model with influence mechanism in social networks. Soft Computing, 1-10.

[19] Zhao, L., Wang, J., Chen, Y., Wang, Q., Cheng, J., \& Cui, H. (2012). SIHR rumor spreading model in social networks. Physica A: Statistical Mechanics and its Applications, 391(7), 2444-2453.

[20] Yang, A., Huang, X., Cai, X., Zhu, X., \& Lu, L. (2019). ILSR rumor spreading model with degree in complex network. Physica A: Statistical Mechanics and its Applications, 531, 121807.

[21] Tran Son Hai, Le Hoang Thai, Nguyen Thanh Thuy,"Facial Expression Classification Using Artificial Neural Network and KNearest Neighbor", International Journal of Information Technology and Computer Science(IJITCS), vol.7, no.3, pp.27-32, 2015. DOI: 10.5815/ijitcs.2015.03.04

[22] Saptarsi Goswami, Amlan Chakrabarti,"Feature Selection: A Practitioner View", International Journal of Information Technology and Computer Science(IJITCS), vol.6, no.11, pp.66-77, 2014. DOI: 10.5815/ijitcs.2014.11.10

\section{Authors' Profiles}

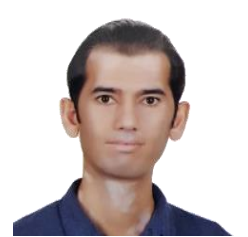

Adel Angali received his B.E. degree in computer software engineering from Lian Institute, Bushehr, Iran, and Department of Computer Engineering in 2011, and has received his M.Sc. degree in computer software engineering from of Lian Bushehr Institute, Iran, in 2021. His hobbies are Graphic Design, Computer Architecture, Computer Communications (Networks), Computer Security and Reliability, and Computer Engineering.

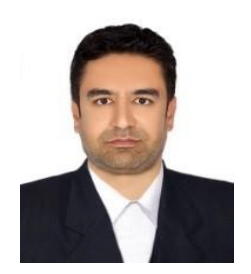

Mousa Mojarad received his PhD in Computer-Software Engineering in 2020. He is currently a lecturer and faculty member of the Islamic Azad University, Firoozabad Branch. His hobbies are Big Data, Face Recognition, Machine Learning, Pattern Recognition and Feature Extraction.

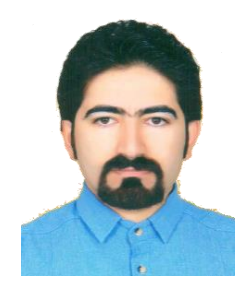

Hassan Arfaeinia obtained her B.E in Computer Science from Rafsanjan University, Kerman, Iran in 2009. HE received her M.S in Computer Engineering from the Amirkabir University of Technology, Tehran, Iran in 2011 and $\mathrm{PhD}$ in Computer Engineering from Islamic Azad University, North Tehran Branch, Iran in 2016. His main paper interests consist of Software Development, Machine Learning, Statistical Modeling and Computer Programming.

How to cite this paper: Adel Angali, Musa Mojarad, Hassan Arfaeinia, "ILSHR Rumor Spreading Model by Combining SIHR and ILSR Models in Complex Networks", International Journal of Intelligent Systems and Applications(IJISA), Vol.13, No.6, pp.51-59, 2021. DOI: $10.5815 /$ ijisa.2021.06.05 